\title{
NFC and VLC based Mobile Business Information System for Registering Class Attendance
}

\author{
Sergio Rios-Aguilar ${ }^{1,2 *}$, Iñigo Sarría ${ }^{3}$, Marta Beltrán Pardo ${ }^{4}$ \\ ${ }^{1}$ Universidad Rey Juan Carlos (URJC), Madrid (Spain) \\ ${ }^{2}$ Universidad Politécnica de Madrid (UPM), Madrid (Spain) \\ ${ }^{3}$ Universidad Internacional de La Rioja (UNIR), Logroño (Spain) \\ ${ }^{4}$ Universidad Rey Juan Carlos (URJC), Madrid (Spain)
}

Received 20 March 2020 | Accepted 29 April 2020 | Published 4 May 2020

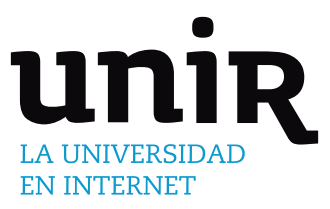

\section{ABSTRACT}

This work proposes a Mobile Information System for class attendance control using Visible Light Communications (VLC), and the students' own mobile devices for automatic clocking in and clocking out. The proposed information system includes (a) VLC physical infrastructure, (b) native Android and iOS apps for the students, and (c) a web application for classroom attendance management. A proof of concept has been developed, setting up a testbed representing a real-world classroom environment for experimentation, using two VLC-enabled LED lighting sources. After three rounds of testing $(n=225)$ under different conditions, it has been concluded that the system is viable and shows consistent positive detections when the smartphones are on the classroom desk within non-overlapped areas of the light circles generated by the LED lighting sources on the table surface. The performed tests also show that if mobile devices are placed within those overlapping areas, the likelihood of a detection error could increase up to nearly $10 \%$, due to multipath effects, and actions can be taken should it happen. Finally, it has to be highlighted that the proposed autonomous class attendance system allows lecturers to focus on making the most of their time in class, transferring knowledge instead of spending time in attendance management task.

\section{KEYWORDS}

Class Attendance, Visible Light Communications, NFC, Presence Information Systems.

\section{INTRODUCTION}

$\mathrm{N}$ the traditional on-site class learning model, student attendance rates have been positively linked in a consistent manner to an increase in academic performance. This attendance is also encouraged by lecturers, which routinely maintain daily records of students attending classes. The attendance recording process, by means of roll call, can be easily unfeasible in large classes, wasting valuable teaching time.

Also, transferring the responsibility of logging their own attendance to the students, passing out a sign-in sheet and collecting it at the end of class may open the possibility of fraud, as absent students could ask other classmates to mark them as "present", and this impersonation can only be detected by means of doing a headcount of the students, which, once again, can take significant time and is prone to error, especially in large classrooms [1],[2].

Therefore, it would be very advisable to devise a highly autonomous class attendance system so that faculties could focus on making the most of their time in class transferring knowledge instead of spending time in attendance management tasks. Also, as the proposed system should be available in every single classroom of the involved teaching institution, the overall cost is an important metric to be considered.

This paper proposes an innovative information system for class attendance management, using Visible Light Communications (VLC) and a mobile app that meet the aforementioned requirements,

\footnotetext{
* Corresponding author.

E-mail address: sj.rios@alumnos.urjc.es
}

minimizing costs and also providing every stakeholder involved with a seamless experience.

The rest of the paper is organized as follows: Section II provides an overview of the related work on class attendance systems and the background on enabling technologies, specifically VLC and NFC, discussing their adequacy for the proposed platform. Section III presents the proposed system. Section IV presents the testbed and methods used for experimentation, the results obtained for validation and evaluation of the proposed system, and discussion of these results. Finally, Section V summarizes conclusions, as well as opportunities and future work.

\section{Related Work And Technology Background}

\section{A. On Class Attendance Systems}

Several research works have been published related to class attendance recording employing biometric systems, such as fingerprint verification, speech recognition and face recognition [3]-[7].

Systems based in fingerprint verification usually involve high costs, as it is necessary to install a reader in every classroom where attendance control is needed. Also, a single reader does not allow multiple simultaneous verifications so it could lead to potential jams, queues and/or delays in the process.

On the other hand, current non-fingerprint based biometric methods for registering attendance are considered too intrusive and sometimes stressful. For example, [8] acknowledges this problem and proposes methods to make the attendance recording process stress-free 
using face biometrics. The system just had $70.83 \%$ accuracy during verification when facial expressions were varied along with variations in lighting conditions during enrollment, and thus the process is still stressful for participants. [2] performs several experiments showing that the deflection angles of faces have severe impacts in the process of face identification. Almost no one of the algorithms evaluated could identify the faces with big pitches, roll or yaws.

In a broad sense, low-cost technology is a general requirement for this kind of systems in the academic world, in order to allow the educational institution to provide all students with it, or to take advantage of technology the students may already be in possession of. It should be a secure technology, additionally, which would reduce insofar as possible, any attempt of fraud (i.e. impersonation) [9],[10]

Also, instead of forcing the student to interact directly with a control device in the classroom, the use of wireless technologies is a usual requirement. Several wireless technologies are considered in research works:

- Bluetooth: It is a rather widespread wireless technology based on a radio frequency that uses the ISM (Industrial, Scientific \& Medical) free radio band on the $2.4 \mathrm{GHz}$. One of the main advantages of this technology is its widespread in current mobile devices. However, a successful exchange of data does not require that emitting and receiving devices are in the same room, so it is not possible with this technology to prove presence at a classroom [9].

- Radio-Frequency Identification tags (RFID): the student would be issued a personal RFID card, and a card reader would be placed in the classroom, which would send data to the server. Thus there is a need to install a device in the classroom with expensive deployment costs, and, as in the case of fingerprint readers, the time needed for clocking-in could lead to queues. Not to mention the difficulty of preventing impersonation frauds.

- Near Field Communication (NFC): This short-range wireless technology is a promising alternative to the abovementioned technologies in class attendance systems, mainly due to the low cost of NFC tags and the increasing availability of the technology in smartphones. One successful approach is described in [9], but the described clock-in process does require the students to tap an NFC card at the beginning of the class, creating a bottleneck and crowds in large classrooms, and also this approach does not prevent early dropouts.

\section{B. On NFC Technology}

Near-field communication (NFC) is a radio-frequency identification system (RFID) that enables devices to communicate in close proximity of around $4 \mathrm{~cm}$, and operates at the High Frequency (HF) band of 13.56 $\mathrm{MHz}$, using electromagnetic induction between two loop antennas. The information exchanged is stored in tiny microchips, called Tags in this domain. These tags can be used over a wide range of materials, such as walls in smart posters, glass, ceramic coffee mugs, plastic and, using a special design, over metals [35],[36].
NFC technology is very convenient and easy to use, enabling users to access digital content or perform contactless transactions with a single tap. Most current smartphones are NFC-enabled and support three operation modes:

- NFC reader/writer, in which an NFC-enabled smartphone acts as an initiator device, generating an RF field, and the target device -inexpensive NFC tags- uses that electromagnetic field to power itself for communication, and then transmits some information back to the smartphone.

- NFC card emulation, in which smartphones behave as contactless smart cards, allowing the users to make payments.

- NFC peer-to-peer, in which a smartphone can exchange information directly with another smartphone, creating an ad-hoc link via WiFi or Bluetooth pairing.

To ensure interoperability when different smartphones retrieve information from a passive tag, the NFC Forum defined a standard data format: NDEF (NFC Data Exchange Format), allowing the use of different types of information: plain text, uniform resource identifiers (URIs), location data, vCard, etc. [30].

\section{On VLC Technology}

Over the last 15 years, the overwhelming development of lightemitting diodes (LEDs) and LED lighting has been one of the major revolutions in the energy sector, mainly thanks to (i) lighting technology development and (ii) global policies put into force worldwide in favour of renewable energy and energy efficiency (LEDs are very environmental-friendly, with reduced emission of $\mathrm{CO} 2$ and with no toxic elements, like mercury) [11],[12].

In fact, LEDs have not only very high efficiency (in terms of energy consumption LEDs can save up to $85 \%$ compared to classic incandescent lamps, and up to $60 \%$ in the case of fluorescents lamps, on an equal basis of brightness and illumination power) but also a long life span (they are expected to last several years), making them ideal for lighting everywhere [13],[14].

One crucial characteristic of LEDs is that visible light (wavelengths from about 380 to 740 nanometers) is harmless to humans (there are no health risks identified distinctly from those of current lighting systems), unlike Infrared (IR) and lasers.

Now, LEDs are also the key pieces that make possible a completely new kind of indoor wireless communications. The light emitted from white lighting LEDs not only can provide illumination, but also can carry binary information at the same time, by means of switching $\mathrm{ON}$ and $\mathrm{OFF}$ at high frequencies (this is the basis of Visible Light Communications, VLC).

In addition, since LEDs modulation is quite easy and straightforward, it is possible to integrate VLC systems into previous LED lighting-only infrastructure. Finally, in terms of spectrum usage, in VLC it is fully unrestricted, unlike RF, whose spectrum is highly regulated for both military and civil use.

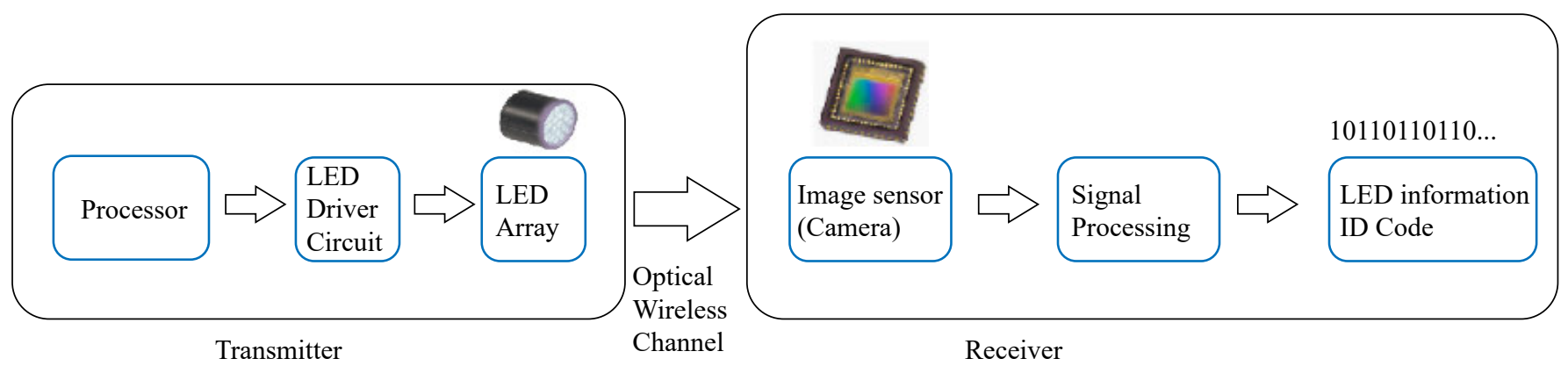

Fig. 1. VLC system components for Optical Camera Communications (OCC). 
In the context of VLC as optical wireless communications, we can find two main system approaches: image sensor based VLC and photodiode-based systems. In the first case, the light is emitted by a LED and is detected using an image sensor, for example, a camera available in a consumer electronic device, and this case is also known as Optical Camera Communications (OCC) [15],[16]. In the second case, the detection is made using a photodiode, a very low-cost device with high sensitivity, but due to its own nature as a discrete semiconductor device, it has to be part of custom-made hardware designs (i.e. not easily available to users like other ubiquitous and commonplace consumer electronic devices) [17],[18].

Today, most of the use cases of VLC are based in two key elements. First, the interference-free nature of the light frequency range, and second, the optical communication takes place exclusively within the cone of light.

As shown in Fig. 1, when a LED transmits the binary information of its own ID Code, using On-Off Keying modulation (OOK), the image sensor captures a series of images for further processing, using a wide range of available algorithms (pixel detection, edge detection, centre of colour balance, etc.) in order to evaluate the $\mathrm{ON}$ or OFF status to extract the ID Code frame [19].

In the past few years, many research works on VLC have been published; most of them focused on the study of system design, medium access and channel characteristics [20]-[22]. Other research works focus on VLC applications, with a high proportion of them related to indoor positioning (knows as Visible Light Positioning, VLP) [11][19][33] as an alternative to other indoor positioning systems based on WiFi, sound or ultrasound or Bluetooth which show lack of accuracy due to the multipath propagation and radio frequency (RF) signal interference. These VLP-related proposals have a common issue: they usually involve high costs and unnecessary complexity, just for the burden of knowing the precise location of the user [34]. In this case, the need is less complex and limited to provide a system with proof of presence of a sensor under a given VLC LED lamp, and thus those costs are not justified. The work [23] surveyed smart lighting and optical wireless communication based on smartphones. As far as the authors know, at the moment of writing this proposal no research work facing the problem of class attendance recording using VLC has been published.

On the basis of the above background, this research work proposes the usage of VLC for class attendance recording, in the form of Optical Camera Communications, using image sensors -cameras- available in smartphones and a native app, avoiding the costs and complexity of a VLP platform.

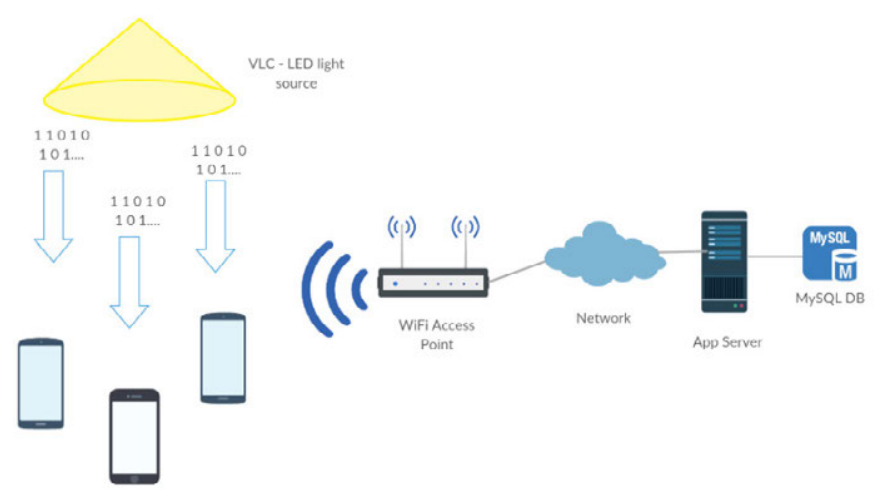

Fig. 2. Proposed System's architecture, including LED lighting sources, Server Application, and mobile phones running a native App.
III. Proposed System

Before describing the relevant components of the proposed system, it is necessary to point out several issues and assumptions made:

- In order to comply with health safety regulations, in particular, to avoid potential epileptic seizures, it has been verified that the modulation of LED lights used is set at a minimum of $1 \mathrm{MHz}$, rendering the visible light generated as flicker-free (over 4.000 times higher than the top-notch $240 \mathrm{~Hz}$ refresh rate found in current professional high-end monitors). So, it is assumed that the LED switching is too fast and not perceivable by humans or animals [24].

- It was decided that the end-users of the project should be the students themselves. Thus, the workload and responsibility of the lecturer are reduced as he would not have to know each and every student from their first day in class, nor keep an eye on who is missing, and can focus completely on teaching the syllabus [9].

The proposed system is comprised of (a) VLC physical infrastructure, (b) native Android and iOS apps for the students, and (c) a server-based web application for classroom attendance management, as shown in Fig. 2.

The workflow in the proposed information systems is as follows:

1. The mobile phones of the students involved must be registered in the class attendance management web application, with proof of identity. This is a one-time in-person onboarding process, common for all of the courses in which the student has enrolled, and it is carried out using NFC.

A central service at the University (or Department depending on the system scale) checks the real identity of the students in-situ, showing them a pre-programmed NFC card so that the students can tap it using their mobile phones, after starting the classroom attendance app. The NFC Card shown to the students can be replaced with a different one -containing a different NDEF message, known to the class attendance management web application- daily (See Fig. 3).

2. The classroom attendance app then reads an encoded text string found in the NFC card and allows the students to complete the registration process. The app sends out both the encoded text received and a unique device identifier (ANDROID_ID or Apple's identifierForVendor), as well as other personal data (Name, Surname and Student_ID).

Before completing this registration process, the students must agree with terms of service, agreeing to abide by the University rules against academic dishonesty specifically related to this classroom attendance system (i.e. impersonation, usage of multisim technology in dual-sim mobile phone, etc.).

3. The management web application keeps relational information about classes, classrooms, students, LED infrastructure in the classroom (VLC IDs), and WiFi Access Points reachable at every classroom (BSSIDs).

4. The students start the classroom attendance app and get into the classroom, placing their mobile phones upwards over the table/ desk, with the front camera facing the ceiling. A connection to the local WiFi access point is required (the BSSID).

5. The app grabs from the server information about lecture times and random-generated times for automatic clocking-in within time margins specified by faculty members. There will be at least two checks for attendance during each class session, one at the beginning (to prevent lateness) and other at the end (to prevent dropouts). So, let's say the initial margin is defined between [ $+5 \mathrm{~min},+15 \mathrm{~min}]$ after the scheduled beginning of the class. The server randomly forces a check for attendance within the initial 


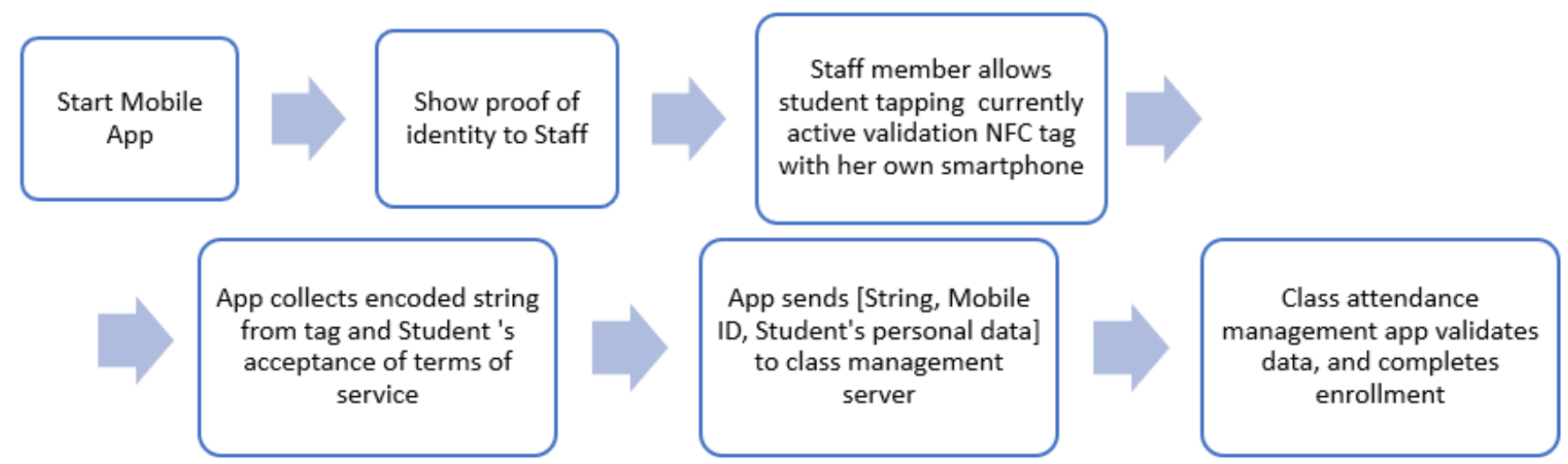

Fig. 3. Flow of the enrollment process (Steps 1-2 in the text), in which the physical validation of the student's identity is carried out offline, followed by an online logical validation.

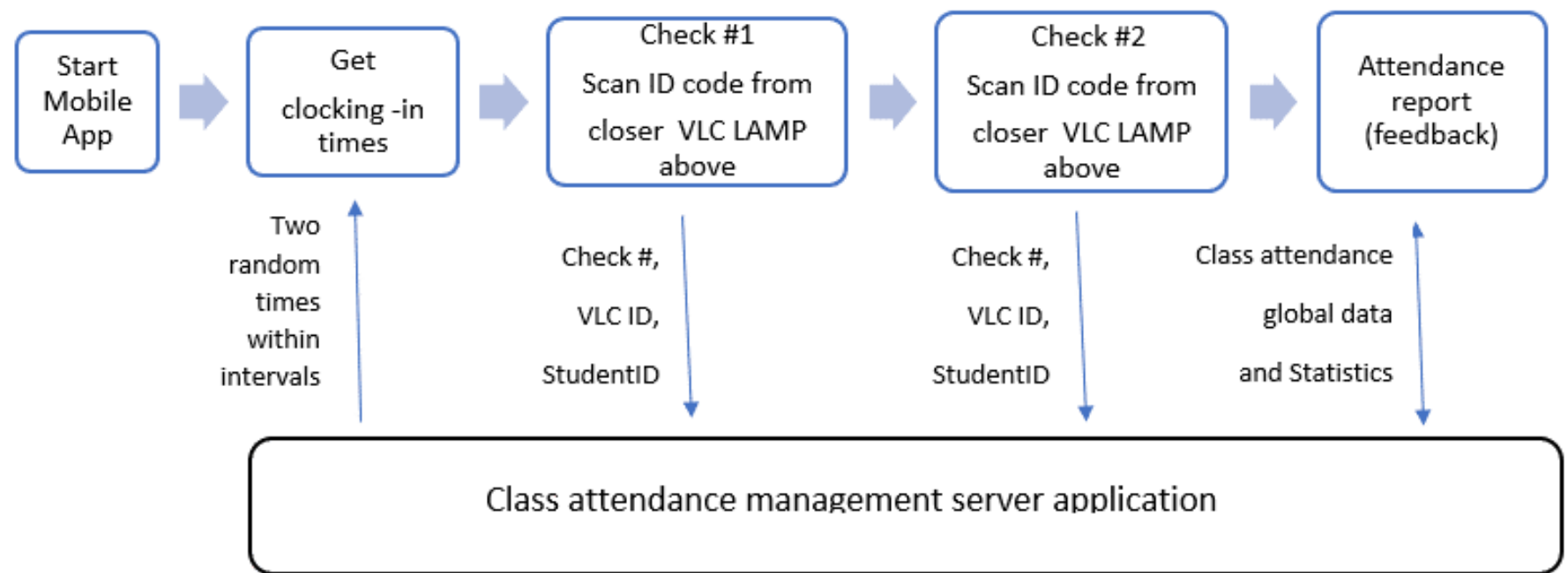

Fig. 4. Flow of operations for registering class attendance in the proposed system, showing the main interactions between the student's mobile app and the class attendance management Web application (Steps 3 to 9 in the text).

margin, for example at +11 min (See Fig. 4).

6. The app, at this specific time, activates the front camera sensor of the mobile phone and scans for the ID code of the VLC lamp more closely located above. The transmitted IDs are decoded through the extraction of the bright and dark stripes of modulated LEDs, using the barcode detection algorithm [25].

7. The app sends the decoded ID, the check number, and a timestamp to the server over an encrypted channel using SSL/TLS, and then the server app validates those data and logs the results (PresenceCheckOK, PresenceCheckNotOK) in the database, associating them with the student ID.

8. The app will provide the student with feedback at the scheduled end of class, be it the time spent attending class, or other results (being late but with correct clocking-out, or clocking-in but without a corresponding clock-out).

9. The faculty member can get attendance statistics by using the management class attendance web application.

Regarding security, some of the aforementioned procedures have been included in the proposed system in order to prevent different forms of fraud of impersonation: checking the BSSID of WiFi's access points, using random times for clocking-in and clocking-out and relying on mobile phones' OS-level Unique IDs, mainly.

Also, VLC systems benefit from immunity to radiofrequency (RF) interference, and by the own nature of VLC technology, all types of possible sources of interferences must be in the visible spectrum, and thus they can be easily spotted by humans. This makes Denial-ofService (DoS) attacks to the LEDs infrastructure easily detectable.

As for the mobile app, there exists the possibility of a DoS attack to the WiFi infrastructure trying to render this kind of required data communications unusable. In fact, DoS attacks based on jamming are really easy to perform on wireless networks, even without sophisticated hardware appliances [32].

But, once again, this kind of attack can be easily detected as it affects a wide range of communication services at the University campus other than the required by the mobile app, so lecturers and other staff can be aware of the problem very early, and act accordingly.

\section{VALIDATION AND Evaluation}

\section{A. Testbed and Methods}

In order to evaluate the proposed system, a testbed has been set up representing a typical classroom environment for two students, which includes a desk table for two students, and two VLC light fixtures (see Fig. 5). Table I shows the detailed arrangements for these elements.

Uniform lighting coverage has been assumed in this work, with purposely generated overlapping light cones over the table, and thus this proposed system will use wide Field-of-View LEDs (with beam angles $\geq 45^{\circ}$ ). This mode of operation has been chosen due to its closer 
resemblance to the real lighting conditions found in classrooms, and also due to the greater complexity of this mode compared to simple spotlighting (discrete and non-overlapping light beams).

Given the beam angle and height of the lamps over the desk table, each light cone intersects the surface of the table resulting in a light circle with a radius $\mathrm{r}=0.86 \mathrm{~m}$. Given the dimensions of the table, this resulting radius makes sure the lights radiate over the full surface of the table. The distance between $100 \%$ light intensity points on the table radiated from both LEDs is $\mathrm{d}=1.5 \mathrm{~m}$.

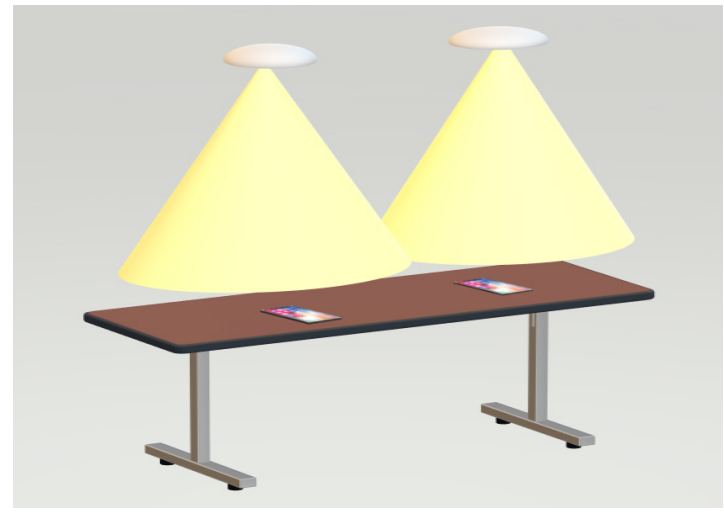

Fig. 5. Representation of the testbed used for the experiments, with one large classroom table and two VLC-enabled LED lighting sources.

TABLE I. Elements Used in the Testbed, with Placement Information AND OTHER DESCRIPTIVE ITEMS

\begin{tabular}{cc}
\hline Element & Data \\
Room size $(\mathrm{m})$ & $4.00(\mathrm{~W}) \times 5.00(\mathrm{D}) \times 2.80(\mathrm{H})$ \\
Number of lamps & 2 \\
Individual LED optical power & $3 \mathrm{~W}$ \\
LED beam angle & 60 degrees \\
LED light color & Warm white $-3000 \mathrm{~K}$ \\
& $(1.25,1.50,1.70)$ and \\
Lamp locations $(\mathrm{m})$ & $(2.75,1.50,1.70)$ \\
Mobile phone locations $(\mathrm{m})$ & $([1.10-2.00],[1.10-1.60], 0.70)$ \\
Desk table dimensions $(\mathrm{m})$ & $2.00(\mathrm{~W}) \times 0.70(\mathrm{D}) \times 0.70(\mathrm{H})$ \\
\hline
\end{tabular}

Both light circles overlap -on purpose for testing- in the centre of the table, and the intersection area can be computed as follows:

$$
A_{\text {intersection }}=2 r^{2} \cos ^{-1}\left(\frac{d}{2 r}\right)-\frac{1}{2} \sqrt{d^{2}(2 r-d)(2 r+d)}
$$

Using the provided values of $\mathrm{r}$ and $\mathrm{d}$ in eq. (1) yields:

$$
A_{\text {intersection }}=1.10 \mathrm{~m}^{2}
$$

This intersection area was marked on the desk table, in order to facilitate the tests in this experiment.

In order to evaluate the proposed system, several tests were made:

- In the first round of tests, the transmitter LEDs were set up to send the same ID Code

- In the second round of tests, the transmitter LEDs were set up to send different ID Codes

- In both rounds of tests, $50 \%$ of them were carried out placing the smartphone in several positions within the non-overlapping area of light circles, and the other $50 \%$ were done correspondingly within that overlapping area.

For every test, what was measured is the detection of the ID code binary pattern using the mobile app. The possible outcomes registered were: Positive Detection (detection with one single scan) and Negative Detection (no detection at all or wrong ID Code detection). Please note that the tests with the mobile phones placed outside the overlapping areas with the same and different IDs are equivalent and therefore have been subsumed into just one case.

The tools used for the measurements were two high-end smartphones running the test app with the following specifications:

- Samsung Galaxy S10, Android version: 9.0 (Android Pie), front camera: 10MP, f/1.9, 26mm, capable of $1080 \mathrm{p}$ at 30 frames per second.

- Apple iPhone XR, iOS version: 12, front camera: 7MP f/2,2, $32 \mathrm{~mm}$, capable of $1080 \mathrm{p}$ at 60 frames per second.

In both cases, the native test app was developed using Oledcomm GEOLiFi API for VLC Communications following the IEEE 802.15.7 standard, using a 32-bits LED lamp ID Code.

\section{B. Results}

The results obtained from the several rounds of tests are summarized in Table II.

TABLE II. Results of the Evaluation of the Proposed System

\begin{tabular}{lccc}
\hline Test & $\mathbf{N}$ & $\begin{array}{c}\text { Positive } \\
\text { Detection }\end{array}$ & $\begin{array}{c}\text { Negative } \\
\text { Detection }\end{array}$ \\
$\begin{array}{l}\text { Samsung/Android smartphone } \\
\text { out of the overlapping area }\end{array}$ & 75 & $100 \%(75)$ & $0 \%$ \\
$\begin{array}{l}\text { Samsung/Android smartphone } \\
\text { within the overlapping area } \\
\text { and Transmitter LEDs sending } \\
\text { same ID Code }\end{array}$ & 75 & $90.7 \%(68)$ & $9.3 \%$ \\
$\begin{array}{l}\text { Samsung/Android smartphone } \\
\text { within the overlapping area } \\
\text { and Transmitter LEDs sending } \\
\text { different ID Code }\end{array}$ & 75 & $92 \%(69)$ & $8 \%$ \\
$\begin{array}{l}\text { Apple iPhone/iOS out of the } \\
\text { overlapping area }\end{array}$ & 75 & $100 \%(75)$ & \\
$\begin{array}{l}\text { Apple iPhone/iOS within } \\
\text { the overlapping area and } \\
\text { Transmitter LEDs sending } \\
\text { same ID Code }\end{array}$ & & & \\
$\begin{array}{l}\text { Apple iPhone/iOS within } \\
\text { the overlapping area and } \\
\text { Transmitter LEDs sending } \\
\text { different ID Code }\end{array}$ & 75 & $93.3 \%(70)$ & $6.7 \%$ \\
\hline
\end{tabular}

\section{Discussion}

After an initial analysis of the obtained results, it seems clear that the barcode OCC detection algorithm is working seamlessly when both types of smartphones are placed within the non-overlapping and interference-free areas of the light circles on the table, (as if the mode of operation had been pure spotlighting from the start).

The problems arise when placing the smartphones within the overlapping area of the light circles on the table. This was somehow expected as in Single Frequency Networks (SFN) structures there are usually severe multipath effects, resulting in inter-symbol interferences [28], [29].

In effect, the rate of negative detections is a bit high, ranging from $6.7 \%$ to $9.3 \%$. In any case, such multipath effects will only appear in the overlapping areas, due to the usually good spatial directivity of light in the visible spectrum [27].

Anyway, the consistent behaviour of the results when using both smartphones within the overlapping area is noteworthy, regardless the use of equal or different LED ID codes ( 5 to 7 false positives in absolute terms in all cases). 
As shown in Table II, the results are slightly better when the LED lamps transmit the same binary ID code, and the iPhone device is used. One possible explanation for this may be related to the image sensor size in the iPhone's front camera, with $23 \%$ more surface available and doubling the sampling rate than those available in the Samsung Galaxy, and both elements contribute to better behaviour of the OCC detection algorithm when inter-symbol interferences are present.

There are several options to compensate the behaviour of the proposed system in real conditions in a classroom: (i) provide the students with visual feedback of the clock-in process, suggesting them to move the smartphone to a more suitable place on the table in case of error (this could be done when they start the class attendance app), (ii) surveying the uniform lighting conditions of the classroom before using the system and adjusting the usually dimmable LED light fixtures trying to minimize the overlapping areas of the light cones at the tables' height.

\section{Conclusions And Future Work}

This work proposes an information system for class attendance control using Visible Light Communications (VLC), using the students' own mobile phone devices for automatic clocking in and clocking out.

A complete flow of operations has been described, and a proof of concept has been developed, setting up a testbed representing realworld classroom environment for experimentation. After three rounds of testing ( $\mathrm{n}=225)$, it has been concluded that the system is viable and works seamlessly when the smartphones are on the desk table within non-overlapped areas of the light circles generated by VLC-enabled LED lighting sources on the table surface. The tests performed also show that if the mobile devices are placed within the aforementioned overlapping areas, the likelihood of a detection error could increase up to nearly $10 \%$ (for the specific smartphone makes and models used in the tests), due to multipath effects.

Therefore, it is essential to minimize those multipath effects by (a) reducing the intersections of the light cones at the height of the classroom desks via local dimming of the light sources without affecting the uniform lighting and/or (b) providing the students with an initial setup of the classroom attendance app to check the adequacy of the current placement of their smartphones on the table.

The proposed information system for class attendance control is practical and brings benefits to both faculty staff and students, resulting in greater control for the educational institution, with fewer management burdens and obligations for lecturers, and additionally providing the students with higher quality teaching with a clear increase in actual teaching time.

One limitation of this work refers to the use of just two smartphones for testing, and that the chosen makes and models are very high-end devices at present. It is needed to perform more intensive testing using a wider range of mobile devices, representing the variety and diversity of student-owned smartphones.

Regarding future works, one interesting idea to develop could be an integration of the modulation circuits at the LED sources with the application server, so that the class attendance application could change on a per-class basis the ID Codes being transmitted, thus making more difficult potential impersonations based in reply-attacks.

Another future line of work will involve the study of the impact in positive detections at overlapping areas if LED sources are organized in a cell system where adjacent LED sources used slightly different frequencies (i.e. colour temperatures) not distinguishable by the human eye.

\section{AUTHOR CONTRIBUTIONS}

Sergio Rios-Aguilar: Conceptualization, Methodology, Writingoriginal draft, Software, Design of Experiment, Data acquisition and analysis, Validation.

Iñigo Sarría: Data Analysis (Review of numerical calculations).

Marta Beltran Pardo: Conceptualization, Writing - Review \& Editing, Supervision.

\section{REFERENCES}

[1] E. Garcia, H. Rivera, N. Ponder, R. Kuo, and J. Zheng, "Efficient and costeffective class attendance management with a smartphone-based system," presented at the Society for Information Technology \& Teacher Education International Conference, 2017, pp. 965-972.).

[2] J. He, Y. Zhao, B. Sun, and L. Yu, "Research on video capture scheme and face recognition algorithms in a class attendance system," presented at the Proceedings of the International Conference on Watermarking and Image Processing, 2017, pp. 6-10.

[3] S. Rao and K. J. Satoa, "An attendance monitoring system using biometrics authentication," International Journal of Advanced Research in Computer Science and Software Engineering, vol. 3, no. 4, pp. 379-383, 2017.

[4] T. Nawas, S. Pervaiz, A. Korrani, and Azhar-ud-din, "Development of academic attendance monitoring system using fingerprint identification," International Journal of Computer Science and Network Security, vol. 9, no. 5,2009 .

[5] Y. Kawaguchi, T. Shoji, W. Lin, K. Kakusho, and M. Minoh, "Face recognition-based lecture attendance system", Department of Intelligence Science and Technology, Graduate School of Informatics, Kyoto University, 2009.

[6] H. Zaman, J. Hossain, T. Anika and D. Choudhury. "RFID based attendance system", 8th International Conference on Computing, Communication and Networking Technologies (ICCCNT), 2017.

[7] S. Dey, S. Barman, R. Bhukya, R. Das, B. Haris, S. Prasanna and R. Sinha, "Speech biometric based attendance system", 2014 Twentieth National Conference on Communications (NCC), 2014.

[8] K. Okokpujie, E. Noma-Osaghae, S. John, K.-A. Grace, and I. Okokpujie, "A face recognition attendance system with GSM notification," presented at the 2017 IEEE 3rd International Conference on Electro-Technology for National Development (NIGERCON), 2017, pp. 239-244.

[9] M.J.L. Fernández, J. Fernández, S. Rios-Aguilar, B. Selvi, and R.G. Crespo. "Control of attendance applied in higher education through mobile NFC technologies". Expert systems with applications, vol. 40, no. 11, pp. 4478-4489, 2013.

[10] S. Rios-Aguilar, J. Pascual-Espada, and R. González-Crespo. "NFC and Cloud-Based Lightweight Anonymous Assessment Mobile Intelligent Information System for Higher Education and Recruitment Competitions". Mobile Networks and Applications, vol. 21, no. 2, pp. 327-336, 2016.

[11] J. Lian, Z. Vatansever, M. Noshad, and M. Brandt-Pearce. "Indoor visible light communications, networking, and applications". Journal of Physics: Photonics, vol. 1, no. 1, p. 012001, 2019.

[12] C. Jurczak. "Review of LiFi visible light communications: research and use cases". Lucibel White Paper. arXiv preprint arXiv:1802.01471, 2017

[13] M. Figueiredo, C. Ribeiro, A. Dobesch, L. N. Alves, and O. Wilfert, "Consumer LED lamp with ODAC technology for high-speed visible light communications," IEEE Trans. Consumer Electron., vol. 63, no. 3, pp. 285-290, August 2017.

[14] A. Jovicic, J. Li, and T. Richardson, "Visible light communication: opportunities, challenges and the path to market". IEEE Commun. Mag., vol. 51, no. 12, pp. 26-32, December 2013.

[15] D. Ganti, W. Zhang and M. Kavehrad. "VLC-based indoor positioning system with tracking capability using Kalman and particle filters." In 2014 IEEE International Conference on Consumer Electronics (ICCE), IEEE, pp. 476-477, January 2014.

[16] M. Yoshino, S. Haruyama and M. Nakagawa. "High-accuracy positioning system using visible LED lights and image sensor." In Radio and Wireless Symposium, IEEE, pp. 439- 442, January 2008.

[17] T.H., Do, M.Yoo. "TDOA-based indoor positioning using visible light". 
Photonic Netw. Commun. no. 27, 2014

[18] Sari Yamaguchi, Vuong V. Mai, Truong C. Thang and Anh T. Pham. "Design and Performance Evaluation of VLC Indoor Positioning System using Optical Orthogonal codes. In 2014 IEEE Fifth International Conference on Communications and Electronics (ICCE), pp. 54-59, IEEE, 2014

[19] N. U. Hassan, A. Naeem, M.A. Pasha, T. Jadoon and C. Yuen. "Indoor positioning using visible led lights: A survey". ACM Computing Surveys (CSUR), vol. 48, no.2, p. 20, 2015

[20] D. Karunatilaka, F. Zafar, V. Kalavally, and R. Parthiban, "LED Based Indoor Visible Light Communications: State of the Art," IEEE communications surveys and tutorials, vol. 17, pp. 1649-1678, 2015

[21] P. H. Pathak, X. Feng, P. Hu, and P. Mohapatra, "Visible Light Communication, Networking, and Sensing: A Survey, Potential and Challenges," IEEE Communications Surveys \& Tutorials, vol. 17, pp. 2047-2077, 2015.

[22] M.A. Khalighi and M. Uysal, "Survey on free space optical communication: A communication theory perspective," IEEE Communications Surveys \& Tutorials, vol. 16, pp. 2231-2258, 2014.

[23] A. Sevincer, M. Bhattarai, M. Bilgi, M. Yuksel, and N. Pala, "LIGHTNETs: Smart LIGHTing and mobile optical wireless NETworks-A survey," IEEE Communications Surveys \& Tutorials, vol. 15, pp. 1620-1641, 2013.

[24] H. Haas. "A light-connected world”. Physics World, vol. 29, no. 8, p. 30, 2016.

[25] C. Fu, C. W. Cheng, W. H. Shen, Y. L. Wei and H. M. Tsai, "Lightbib: marathoner recognition system with visible light communications," IEEE Int. Conf. Data Science and Data Intensive Sys., pp. 572-578, 2015.

[26] Y. Li, Z. Ghassemlooy, X. Tang, B. Lin, and Y. Zhang, "A VLC smartphone camera based indoor positioning system”. IEEE Photonics Technology Letters, vol. 30, no.13, pp.1171-1174, 2018.

[27] J. Song, W. Ding, F. Yang, H. Yang, B. Yu, B. and H. Zhang. "An indoor broadband broadcasting system based on PLC and VLC". IEEE Transactions on Broadcasting, vol. 61, no. 2, pp.299-308, 2015.

[28] R. Kaur and S. Arora. "Nature Inspired Range Based Wireless Sensor Node Localization Algorithms". International Journal of Interactive Multimedia \& Artificial Intelligence, vol. 4, no. 6, pp. 7-17, 2017.

[29] M. Nighot, A. Ghatol and V. Thakare. "Self-Organized Hybrid Wireless Sensor Network for Finding Randomly Moving Target in Unknown Environment". International Journal of Interactive Multimedia \& Artificial Intelligence, vol. 5, no. 1, pp.16-28, 2018.

[30] A. Lesas and S. Miranda. "The art and science of NFC Programming". Hoboken, NJ: Jonh Wiley \& Sons, Inc, 2017.

[31] C. Rohner, S. Raza, D. Puccinelli and T. Voigt. "Security in visible light communication: Novel challenges and opportunities". Sensors \& Transducers Journal, vol. 192, no.9, pp. 9-15, 2015

[32] A.D. Wood, J.A. Stankovic and G. Zhou. "DEEJAM: Defeating energyefficient jamming in IEEE 802.15. 4-based wireless networks”. In 2017 4th Annual IEEE Communications Society Conference on Sensor, Mesh and Ad Hoc Communications and Networks, pp. 60-69. IEEE. June, 2017

[33] D. H. Mai and A. T. Pham. Implementation and Evaluation of VLCBased Indoor Positioning Systems for Smart Supermarkets. In 2018 9th International Conference on Awareness Science and Technology (iCAST), pp. 273-278. IEEE, 2018.

[34] Y. Zhuang, L. Hua, L. Qi, J. Yang, P. Cao, Y. Cao, Y. Wu, J. Thompson and H. Haas. "A Survey of Positioning Systems Using Visible LED Lights”. IEEE Communications Surveys \& Tutorials, 2018. 10.1109/ COMST.2018.2806558.

[35] D. Saeed, R. Iqbal, h.H.R. Sherazi and U.G. Khan.” Evaluating NearField Communication tag security for identity theft prevention". Internet Technology Letters, vol. 2, no. 5, p. e123, 2019

[36] A. Lazaro, R.Villarino, and D. Girbau. "A survey of NFC sensors based on energy harvesting for IoT applications". Sensors, vol. 18, no.11, 3746, 2018.

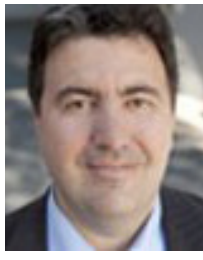

Sergio Ríos Aguilar

Sergio Rios received the Master's degree in Telematics and Computer Systems from Universidad Rey Juan Carlos (Spain). He also holds a $\mathrm{PhD}$ degree (Cum Laude) in Economics (Organization Engineering) from the Department of Management, School of Business and Economics, Universidad de Granada (Spain) in 2013. Dr. Ríos has worked as a consultant in projects for several international companies like Samsung Electronics, Nokia, INTU Group, and others. He is a member of the European Global Navigation Satellite Systems Agency's (GSA) Galileo Raw Measurement Taskforce. His current research interests include Mobile Business Information Systems, Mobile Presence and attendance Systems, Location based Services, Mobile Edge Computing and Internet of Things.

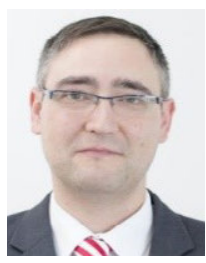

\section{Iñigo Sarría Martínez de Mendivil}

Iñigo Sarria holds a Doctorate (Cum Laude and special prize) from the International University of La Rioja, a Bachelors degree in Mathematics from the University of the Basque Country, a University Expert qualification in Analysis of the Knowledge Society from The International University of La Rioja, and a Certificate in Teaching from the Complutense University of Madrid. He is Associate Vice-Chancellor of Academic Affairs and PDI of the Computer Science and Technology Area of the School of Engineering and Technology at the International University of La Rioja. ORCID code: https://orcid.org/0000-00022584-9671

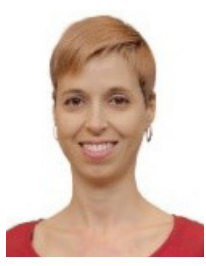

\section{Marta Beltrán Pardo}

Marta Beltrán received the master's degree in Electrical Engineering from Universidad Complutense of Madrid (Spain) in 2001, the master's degree in Industrial Physics from UNED (Spain) in 2003 and the PhD degree from the Department of Computing, Universidad Rey Juan Carlos, Madrid (Spain) in 2005. She is currently working with this department as an Associate Professor. She is the leader of the GAAP research group, co-founder of the Cybersecurity Cluster and she has published extensively in high-quality national and international journals and conference proceedings in the areas of security\&privacy, and parallel and distributed systems. Her current research interests are Cloud computing, Edge/Fog Computing and Internet of Things, specifically, Identity and Access Management and privacy-preserving mechanisms for these paradigms. 